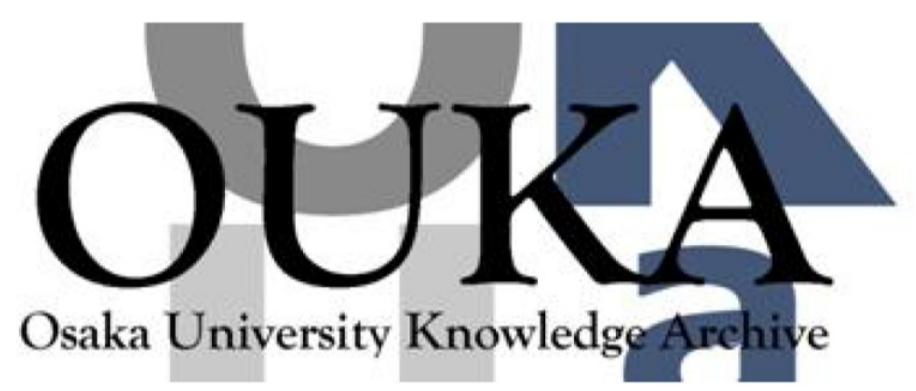

\begin{tabular}{|c|c|}
\hline Title & $\begin{array}{l}\text { A Temperature Responsive Polysaccharide } \\
\text { Derivative in Aqueous Solution : Amylose Ethyl } \\
\text { Carbamates }\end{array}$ \\
\hline Author (s) & $\begin{array}{l}\text { Kimura, Shunji; Kochi, Ryotaro; Kitamura, } \\
\text { Shinichi et al. }\end{array}$ \\
\hline Citation & $\begin{array}{l}\text { ACS Applied Polymer Materials. 2(6) p. 2426- } \\
\text { p. } 2433\end{array}$ \\
\hline Issue Date & $2020-06-12$ \\
\hline oaire:version & AM \\
\hline URL & https://hdl. handle. net/11094/81795 \\
\hline rights & $\begin{array}{l}\text { This document is the Accepted Manuscript } \\
\text { version of a Published Work that appeared in } \\
\text { final form in ACS Applied Polymer Materials, } \odot \\
\text { American Chemical Society after peer review and } \\
\text { technical editing by the publisher. To access } \\
\text { the final edited and published work see } \\
\text { https://doi.org/10.1021/acsapm. Oc00366. }\end{array}$ \\
\hline Note & \\
\hline
\end{tabular}

Osaka University Knowledge Archive : OUKA

https://ir. Library. osaka-u. ac. jp/

Osaka University 


\title{
A Temperature Responsive Polysaccharide Derivative in Aqueous Solution: Amylose Ethyl Carbamates
}

\author{
Shunji Kimura,,$^{\dagger}$ Ryotaro Kochi, ${ }^{\dagger}$ Shinichi Kitamura, ${ }^{+}$and Ken Terao ${ }^{*}+\dot{+}$ \\ †Department of Macromolecular Science, Graduate School of Science, Osaka University, 1-1 \\ Machikaneyama-cho, Toyonaka, Osaka 560-0043, Japan \\ Center for Research and Development of Bioresources, Organization for Research Promotion, \\ Osaka Prefecture University, 1-2, Gakuen-cho, Naka-ku, Sakai, 599-8570, Japan \\ * Corresponding author. Tel.: +81 66850 5459; fax: +8166850 5461. \\ E-mail address: kterao@chem.sci.osaka-u.ac.jp
}

ABSTRACT: Partly substituted amylose ethylcarbamate (AEC) samples for which degree of substitution $D S$ and weight-average molar mass $M_{\mathrm{w}}$ range between $0.21-1.95$ and $40-130 \mathrm{~kg}$ $\mathrm{mol}^{-1}$, respectively, were prepared from enzymatically synthesized amylose. The particle scattering function $P(q)$ of the AEC samples in polar organic solvents and water showed that local helical structure is retained as in the case of amylose and fully substituted AEC. The AEC samples with $D S<1.3$ were soluble in water, and furthermore, the lower critical solution temperature (LCST) type phase separation was found for the samples with $0.9<D S<1.3$. The cloud point temperature $T_{\text {cloud }}$ varied from $12{ }^{\circ} \mathrm{C}$ to $41{ }^{\circ} \mathrm{C}$ depending on $D S, M_{\mathrm{w}}$, and the polymer mass concentration $c$. The $T_{\text {cloud }}$ values determined as a function of $c$ had a minimum at $10-100 \mathrm{mg}$ $\mathrm{mL}^{-1}$. The LCST tended to rise with lowering $M_{\mathrm{w}}$ and $D S$. Iodine test indicated that all watersoluble AEC samples formed complex with iodine while the absorption peak wavelength shifted blue with increasing $D S$. The local helical structure of AEC may form inclusion complex with hydrophobic molecules. AEC is possibly used for a novel capture material which can be separated from water by temperature elevation.

Key Words: polysaccharide derivatives, water-soluble polymers, LCST phase separation, inclusion complex, helical polymers, semiflexible polymers.

\section{Introduction}

The entropy of dissolution for polymer solutions is substantially smaller than those for the small molecules. ${ }^{1}$ Relatively small attractive interactions between polymer molecules can cause the phase separation of the solution. Especially for aqueous solution systems, phase separation phenomena are widely considered for medical applications, such as, tissue culture scaffolds and drug delivery systems. ${ }^{2-6}$ Such polymers are called as temperature-responsive polymers. A number of synthetic temperature-responsive polymers are reported and one of the most prominent polymers is poly $\left(N\right.$-isopropylacrylamide) (PNIPAM),${ }^{7-9}$ for which phase separation temperature is 
tunable by using appropriate random copolymers. ${ }^{10-14}$ Synthetic polymers however have a disadvantage taking the less biodegradability into account. On the other hand, aqueous solutions of natural polysaccharide derivatives are temperature responsive. For example, methylcellulose ${ }^{15}$ is a typical polysaccharide derivative for which aqueous solution has lower critical solution temperature (LCST) and the phase separation behavior is now a hot research topic because higher order structures are found in the concentrated phase. ${ }^{16-17}$ Furthermore, Ju et al. ${ }^{18}$ recently reported that oligoethylene glycol grafted starch is soluble in water and their aqueous solution has phase separation behavior between $17.5^{\circ} \mathrm{C}$ and $55^{\circ} \mathrm{C}$ depending on the grafting density.

According to Burchard, ${ }^{19}$ solutions of carbamate derivatives of cellulose and amylose have phase separation behavior in some organic solvents. Recently, we also found that both LCST and upper critical solution temperature (UCST) systems for amylose carbamates when we chose appropriate side groups and solvents..$^{20-21}$ Furthermore, aqueous solutions of partly substituted polyvinyl alcohol carbamates have solubility and temperature-responsive behavior in water. ${ }^{22}$ It is therefore reasonably expected that aqueous solution of partly substituted amylose alkyl carbamates may be temperature responsive. Indeed, in our preliminary experiments, aqueous solution of amylose ethylcarbamate (AEC) the chemical structure of which is shown in Chart 1 had good solubility in water at low temperature but became clouded with raising temperature, suggesting AEC has LCST in water.

Chart 1. Chemical Structure of AEC.

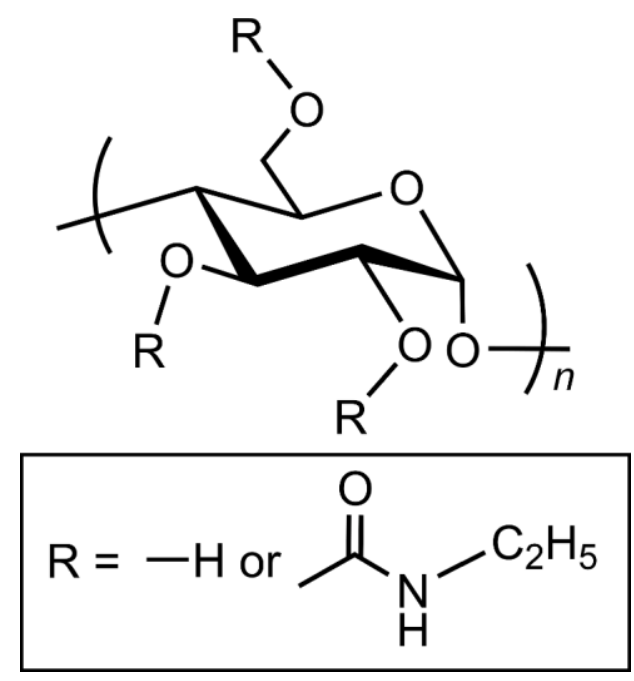

We thus synthesized partly substituted AEC samples for which degree of substitution (DS) ranges between 0.2 and 2 to determine phase diagram of the aqueous solution and to characterize the LCST behavior. The local helical structure and chain stiffness in organic and aqueous solvents were also determined as a function of $D S$ to investigate the helical nature of the polymer. Taking into account the appreciable biodegradability of fatty acid esters of amylose, ${ }^{23}$ partly substituted AEC may be biodegradable. As is well-known, inclusion complex formation of the helical main chain is one of the representative characteristics for starch and amylose. If AEC retains inclusion complex forming ability, it can be applied for capturing materials of hydrophobic materials. Consequently, iodine test was carried out for all water-soluble AEC samples. 


\section{Experimental Section}

Samples. Previously investigated enzymatically synthesized amylose ${ }^{24}$ samples, ESA50K and ESA120K, were used to prepare AEC samples. ${ }^{25}$ The weight-average molar mass $M_{\mathrm{w}}$ and the dispersity index $Ð$ for ESA50K were determined to be $50 \mathrm{~kg} \mathrm{~mol}^{-1}$ and 1.2 from those for the corresponding phenylcarbamate derivative samples. ${ }^{25-26}$ A typical procedure to synthesize an AEC sample is as follows in the manner reported previously. ${ }^{27}$

An amylose sample ESA50K ( $2 \mathrm{~g}, 12 \mathrm{mmol})$ was dried in a two-necked flask in a vacuum at $100{ }^{\circ} \mathrm{C}$ for $3 \mathrm{~h}$ with $\mathrm{LiCl}$ (Fujifilm-Wako, $2 \mathrm{~g}$ ). $N, N$-dimethylacetamide (Wako, super dehydrated grade, $70 \mathrm{~mL}$ ) was added into the flask to dissolve both $\mathrm{LiCl}$ and amylose by stirring at $120{ }^{\circ} \mathrm{C}$. Pyridine $(80 \mathrm{~mL})$ distilled over $\mathrm{CaH}_{2}$ and ethyl isocyanate (Wako, $3.7 \mathrm{~mL}, 39 \mathrm{mmol}$ ) were added to the flask. The mixture was then stirred for $12 \mathrm{~h}$ at $120^{\circ} \mathrm{C}$. The molar ratio of ethyl isocyanate to the hydroxy group of amylose was chosen to be between 0.2 and 1.0 to obtain different $D S$ samples. The reaction mixture was poured into acetone or water to precipitate the AEC sample.

The crude samples were dried in a vacuum at room temperature and purified by the following procedures. Since most samples were soluble in water at room temperature except for the highest $D S$ sample, the water-soluble samples were dissolved in water and dialyzed against water for at least 4 days. The resulting aqueous solutions were lyophilized to obtain the solid samples. A fractional precipitation procedure was employed for a water-insoluble sample with methanol as a solvent and water as a precipitant. The methanol solution of an appropriate middle fraction was poured into water to precipitate the AEC sample. The chemical structure of the purified samples was confirmed by ${ }^{1} \mathrm{H}-\mathrm{NMR}$ in deuterated dimethyl sulfoxide with a small amount of (trimethylsilyl)-1-propanesulfonic acid sodium salt (Figure S1) whereas the intensity of each peak cannot be estimated properly as in the case of our previous study for fully substituted derivatives. ${ }^{27-28}$ The degree of substitution $D S$ for which full substitution is defined as $D S=3$ was determined from the weight ratio of carbon to nitrogen atoms which were determined by ultimate analysis. In the present study, we chose the conventional method to obtain AEC while a modern procedure without hazardous reagents ${ }^{29}$ could be applicable to synthesize low $D S$ samples.

Static and Dynamic Light Scattering in Organic Solvents. Both static light scattering (SLS) and dynamic light scattering (DLS) measurements were made for all AEC samples in $N, N$ dimethylformamide (DMF) at $25{ }^{\circ} \mathrm{C}$ and AEC50K1.95 in methanol at $25{ }^{\circ} \mathrm{C}$ with an ALV/SLS/DLS-5000 light scattering photometer. An Nd: YAG laser with a wavelength $\lambda_{0}=532$ $\mathrm{nm}$ in a vacuum was used as an incident light. Each solution was optically cleaned with a PTFE filter, for which pore size was $0.45 \mu \mathrm{m}$ and was poured into a cylindrical cell with $14 \mathrm{~mm}$ i.d. Distilled toluene was used as the standard sample to evaluate the Rayleigh ratio $R_{q}$ from the scattering intensity where $q$ denotes the magnitude of the scattering vector. Both $R_{q}$ and the autocorrelation function $g^{(2)}(t)$ of the scattering intensity were determined at different $q$. The CONTIN analysis was examined for the $g^{(2)}(t)$ data to estimate apparent hydrodynamic radius $R_{\mathrm{H} \text {,app }}$ and the spectrum $A\left(R_{\mathrm{H} \text {,app }}\right)$. Some $A\left(R_{\mathrm{H} \text {,app }}\right)$ data were bimodal as shown in Figure $\mathrm{S} 2$ in the Supporting information, owing to small amount of large particles consisting probably of associated polymer species. Since the weight fraction of the aggregated species is negligibly small except for AEC120K1.23, the Rayleigh ratio $R_{q, 1}$ from the dispersed polymer (the fast component) was estimated from $R_{q}$ and the area ratio of the $A\left(R_{\mathrm{H}, \mathrm{app}}\right)$ in the manner reported previously. ${ }^{30-31}$ The 
resulting $R_{q, 1}$ [or $R_{q}$ for monomodal $A\left(R_{\mathrm{H}, a p p}\right)$ ] data at different $q$ and at four different polymer mass concentrations $c$ were analyzed in terms of the Berry plot $^{32}$ (the square-root Zimm plot, see Figure S3) to determine the weight-average molar mass $M_{\mathrm{w}}$ and the second virial coefficient $A_{2}$ because the Berry plot has a better linearity than the conventional Zimm plots for semiflexible polymers in dilute solution. Mostly flat $q$ dependence of $K c / R_{q, 1}$ indicates gyration radii of the samples cannot be determined from SLS. It should be noted that the $M_{\mathrm{w}}$ value for AEC120K1.23 was estimated from $M_{\mathrm{w}}$ of AEC120K0.97 assuming the same degree of polymerization. The apparent hydrodynamic radius $R_{\mathrm{H}, 1 \text {,app }}$ for the fast component was extrapolated to $q^{2}=0$ and $c=0$ as displayed in Figure $\mathrm{S} 4$ to determine the hydrodynamic radius $R_{\mathrm{H}, 1}$. The refractive index increments of the AEC samples in solution at $\lambda_{0}=532 \mathrm{~nm}$ were determined with a Schulz-Cantow type differential refractometer. The evaluated values in DMF were between 0.074 and $0.090 \mathrm{~cm}^{3} \mathrm{~g}^{-1}$ depending on $D S$ and that for AEC50K1.95 in methanol was $0.146 \mathrm{~cm}^{3} \mathrm{~g}^{-1}$. It should be noted that light scattering measurements were infeasible for aqueous systems owing to the high scattering intensity from aggregates.

Small-Angle X-ray Scattering in Organic and Aqueous Solvents. SAXS measurements were examined for dilute AEC solutions at BL40B2 beamline in SPring-8 (Hyogo, Japan) or at BL-6A beamline in KEK-PF (Ibaraki, Japan). ${ }^{33}$ The wavelength $\left(\lambda_{0}\right)$ and the camera length were set to be $0.1 \mathrm{~nm}$ and $4 \mathrm{~m}$ for SPring- 8 and $0.15 \mathrm{~nm}$ and $2.5 \mathrm{~m}$ for KEK-PF, respectively. A quartz capillary cell $(2.0 \mathrm{~mm} \phi)$ was installed in a thermostatic cell holder. The scattered light was acquired by Dectris Pilatus 3S 2M (SPring-8) or Pilatus $31 \mathrm{M}$ (KEK-PF) two-dimensional detectors. The $q$ value at each pixel on the detector was estimated by the diffraction from silver behenate. A circular average procedure was employed with the SAngler software ${ }^{34}$ to yield the scattering intensity data as a function of $q$. The scattering intensity was normalized with the intensity of the incident light detected at the lower end of the cell to compensate both intensity of the incident light and the transparency of the solution. The measurement was made for all samples in DMF at $25{ }^{\circ} \mathrm{C}$, AEC50K1.95 in methanol at $25{ }^{\circ} \mathrm{C}$, and AEC50K0.21, AEC50K0.54, and AEC50K0.69 in water at various temperatures from $10{ }^{\circ} \mathrm{C}$ to $50^{\circ} \mathrm{C}$. The data obtained at different $c$ between 1 and $10 \mathrm{mg} \mathrm{mL}^{-1}$ were extrapolated to $c=0$ and $q^{2}=0$ to determine the $z$-average mean-square radius of gyration $\left\langle S^{2}\right\rangle_{z}$ and the particle scattering function $P(q)$ (See Figure S5).

Turbidity of Aqueous Solution. Light transmittance measurement was carried out for AEC50K1.22, AEC50K0.99, AEC120K1.23, and AEC120K0.97 in water with a Jasco V-550 $\mathrm{UV}-\mathrm{V}$ is spectrophotometer to determine the cloud point temperature $T_{\text {cloud }}$ as a function of $c$ ranging between 3 and $210 \mathrm{mg} \mathrm{mL}^{-1}$. Test solution in a rectangular quartz cell with $2 \mathrm{~mm}$ path length was heated with the rate of $0.5^{\circ} \mathrm{C} \mathrm{min}$. Visible light with $\lambda_{0}=550 \mathrm{~nm}$ was used as the incident light.

Differential Calorimetry (DSC) for Aqueous Solution. DSC measurements were made on a TA Instrument Nano DSC for AEC50K1.22, AEC50K0.99, and AEC120K0.97 in water. Polymer mass concentration $c$ was chosen to be between 90 and $120 \mathrm{mg} \mathrm{mL}^{-1}$. The molar concentration of monosaccharide unit calculated from $c M_{0}^{-1}$ to be between $0.40 \mathrm{M}$ and $0.47 \mathrm{M}$, for which average molar mass $M_{0}$ of the repeat unit is $0.248,0.232$, and $0.231 \mathrm{~kg} \mathrm{~mol}^{-1}$ for AEC50K1.22, AEC50K0.99, and AEC120K0.97, respectively. Temperature rising rate was set to be $1{ }^{\circ} \mathrm{C} \mathrm{min}^{-1}$. 
Iodine Test. Since appreciable color change was observed when we added aqueous iodine solution to AEC, iodine test was made for AEC50K1.22, AEC50K0.99, AEC50K0.69, AEC50K0.54, and AEC50K0.21. A small amount $(30 \mu \mathrm{L})$ of aqueous iodine $(0.5 \mathrm{M})$ and potassium iodine $(0.5 \mathrm{M})$ solution was added to $1.5 \mathrm{~mL}$ of aqueous AEC solution $\left(c=5 \mathrm{mg} \mathrm{mL}^{-1}\right)$. The molar concentration $\mathrm{I}_{2}$ in the mixture is calculated to be $10 \mathrm{mM}$. The UV-Vis measurement was carried out for each mixture with the Jasco V-550 UV-Vis spectrophotometer and a quartz rectangular cell of $1 \mathrm{~mm}$ path length. The measurement was done at $25^{\circ} \mathrm{C}$ for the four low $D S$ samples while $10^{\circ} \mathrm{C}$ was chosen for AEC50K1.22 to avoid phase separation.

\section{Results and Discussion}

Molecular Characteristics of AEC in Organic Solvents. The obtained $D S$ values are summarized in Table 1 . These values indicate that $65-90 \%$ of isocyanate molecules were reacted with the hydroxyl groups of amylose except for AEC50K0.22, for which the value was around $10 \%$ probably due to the contamination. As is shown in the table, the water-insoluble sample is AEC50K1.95. Aqueous solutions of the four samples, AEC50K1.22, AEC50K0.99, AEC120K1.23, and AEC120K0.97 became turbid with raising the temperature while the other samples were well soluble in water between 4 and $80{ }^{\circ} \mathrm{C}$. Weight-average molar mass $M_{\mathrm{w}}, A_{2}$, $\left\langle S^{2}\right\rangle_{\mathrm{z}}$, and $R_{\mathrm{H}, 1}$ determined in DMF (or in methanol) are also summarized in the table. The degree of polymerization for AEC50K series are substantially the same or somewhat smaller than that for ESA50K, suggesting chain degradation was not significant. Large positive $A_{2}$ indicates DMF is a good solvent for AEC samples even though amylose is not soluble in the solvent even though the accuracy of $A_{2}$ is not high enough to discuss the $D S$ dependence.

Table 1. Molecular Characteristics of AEC Samples

\begin{tabular}{|c|c|c|c|c|c|c|c|c|c|}
\hline Sample & $D S$ & $\begin{array}{c}M_{\mathrm{w}} \\
/ \mathrm{kg} \mathrm{mol}^{-1, \mathrm{~d}}\end{array}$ & $\begin{array}{c}10^{4} A_{2} \\
/ \mathrm{mol} \mathrm{m}^{3} \mathrm{~kg}^{-2, \mathrm{~d}}\end{array}$ & $\begin{array}{c}\left\langle S^{2}\right\rangle_{z}^{1 / 2} \\
/ \mathrm{nm}^{\mathrm{f}}\end{array}$ & $\begin{array}{l}R_{\mathrm{H}, 1} \\
/ \mathrm{nm}^{\mathrm{e}}\end{array}$ & $\begin{array}{c}h \\
/ \mathrm{nm}\end{array}$ & $\begin{array}{l}\lambda^{-1} \\
/ \mathrm{nm}\end{array}$ & $\begin{array}{c}d \\
/ \mathrm{nm}\end{array}$ & $\begin{array}{c}T_{\mathrm{c}} \\
/{ }^{\circ} \mathrm{C}\end{array}$ \\
\hline АEC50K1.95 & 1.95 & $\begin{array}{l}50^{\mathrm{a}} \\
55^{\mathrm{b}}\end{array}$ & $\begin{array}{l}4.8^{\mathrm{a}} \\
2.5^{\mathrm{b}}\end{array}$ & $\begin{array}{l}7.7^{\mathrm{a}} \\
7.3^{\mathrm{b}}\end{array}$ & $\begin{array}{l}5.5^{\mathrm{a}} \\
4.3^{\mathrm{b}}\end{array}$ & $\begin{array}{l}0.41^{\mathrm{a}} \\
0.37^{\mathrm{b}}\end{array}$ & $\begin{array}{l}6.2^{\mathrm{a}} \\
5.5^{\mathrm{b}}\end{array}$ & $\begin{array}{l}1.7^{\mathrm{a}} \\
1.4^{\mathrm{b}}\end{array}$ & I \\
\hline AEC50K1.22 & 1.22 & $42^{\mathrm{a}}$ & $4.6^{\mathrm{a}}$ & $6.8^{\mathrm{a}}$ & $4.1^{\mathrm{a}}$ & $0.33^{\mathrm{a}}$ & $5.8^{\mathrm{a}}$ & $1.5^{\mathrm{a}}$ & 16 \\
\hline AEC50K0.99 & 0.99 & $71^{\mathrm{a}}$ & $2.4^{\mathrm{a}}$ & $7.8^{\mathrm{a}}$ & $5.4^{\mathrm{a}}$ & $0.24^{\mathrm{a}}$ & $5.5^{\mathrm{a}}$ & $1.0^{\mathrm{a}}$ & 31 \\
\hline АEC50K0.69 & 0.69 & $45^{\mathrm{a}}$ & $8.6^{\mathrm{a}}$ & $7.0^{\mathrm{a}}$ & $4.2^{\mathrm{a}}$ & $0.28^{\mathrm{a}}$ & $5.4^{\mathrm{a}}$ & $1.4^{\mathrm{a}}$ & S \\
\hline АEC50K0.54 & 0.54 & $49^{\mathrm{a}}$ & $7.9^{\mathrm{a}}$ & $6.9^{\mathrm{a}}$ & $4.5^{\mathrm{a}}$ & $0.23^{\mathrm{a}}$ & $5.5^{\mathrm{a}}$ & $1.2^{\mathrm{a}}$ & S \\
\hline AEC50K0.21 & 0.21 & $52^{\mathrm{a}}$ & $2.7^{\mathrm{a}}$ & $6.5^{\mathrm{a}}$ & $4.4^{\mathrm{a}}$ & $0.19^{a}$ & $4.9^{\mathrm{a}}$ & $1.1^{\mathrm{a}}$ & S \\
\hline AEC120K1.23 & 1.23 & $134^{\mathrm{c}}$ & - & $13^{\mathrm{a}}$ & - & $0.31^{\mathrm{a}}$ & $6.3^{\mathrm{a}}$ & $1.3^{\mathrm{a}}$ & 11 \\
\hline AEC120K0.97 & 0.97 & $120^{\mathrm{a}}$ & $4.7^{\mathrm{a}}$ & $13^{\mathrm{a}}$ & $8.3^{\mathrm{a}}$ & $0.33^{\mathrm{a}}$ & $6.0^{\mathrm{a}}$ & $1.3^{\mathrm{a}}$ & 25 \\
\hline
\end{tabular}

${ }^{\mathrm{a}}$ In DMF. ${ }^{\mathrm{b}}$ In methanol. ${ }^{\mathrm{c}}$ Estimated from $M_{\mathrm{w}}$ of AEC120K0.97. ${ }^{\mathrm{d}}$ From SLS. ${ }^{\mathrm{e}}$ From DLS. ${ }^{\mathrm{f}}$ From SAXS. $T_{\mathrm{c}}$ : LCST in water. I: insoluble in water. S: soluble in water between 4 and $80^{\circ} \mathrm{C}$. 
Figure 1 illustrates the Holtzer plot $^{35}$ for the AEC samples in DMF and methanol. The Holtzer plateau can be found around $q=1 \mathrm{~nm}^{-1}$ and a peak is found in the lower $q$ range, indicating the AEC chain is relatively flexible in the solvent. The data were analyzed in terms of the touchedbead wormlike chain for which $P(q)$ can be expressed as ${ }^{36-37}$

$$
\begin{aligned}
& P(q)=9\left(\frac{2}{q d}\right)^{6}\left(\sin \frac{q d}{2}-\frac{q d}{2} \cos \frac{q d}{2}\right)^{2} P_{0}(q) \\
& P_{0}(q)=\frac{2}{L^{2}} \int_{0}^{L}(L-t) I\left(\lambda^{-1} q ; \lambda t\right) \mathrm{d} t
\end{aligned}
$$

where $L, \lambda^{-1}$, and $d$ indicate the contour length, the Kuhn segment length (a measure of the chain stiffness), and the bead diameter, respectively; note that the persistence length is half of $\lambda^{-1}$. The Nakamura-Norisuye expression ${ }^{38-39}$ was utilized to calculate the characteristic function $I\left(\lambda^{-1} q ; \lambda t\right)$ numerically. At least the former two parameters, $L$ and $\lambda^{-1}$, were unequivocally determined by a curve fitting procedure. In actuality, $L$ can be estimated from the height of the plateau around $q=$ $1 \mathrm{~nm}^{-1}$. The parameter $\lambda^{-1}$ can be determined by the difference between the solid curve for the wormlike chain and the dashed line for the rod limit. The accuracy of the last parameter $(d)$ is somewhat worse than the others because $q P(q)$ decreases with increasing $q$ only gradually at high $q$ range. The obtained parameters are also summarized in Table 1, where $h$ is the helix rise (or helix pitch) per residue defined as $h \equiv L M_{0} / M_{\mathrm{w}}$ with $M_{0}$ being the average molar mass of the saccharide unit. It should be noted that intramolecular excluded-volume effects are not significant on the basis of the quasi-two-parameter theory ${ }^{37,40-41}$ since the Kuhn segment number $\lambda L$ is less than 30 for the current samples. ${ }^{42-43}$ The radius of gyration $\left\langle S^{2}\right\rangle^{1 / 2}$ for the wormlike chain can be calculated by the Benoit-Doty equation; ${ }^{44}$ note that the chain thickness effect on $\left\langle S^{2}\right\rangle^{1 / 2}$ is negligible. The calculated $\left\langle S^{2}\right\rangle^{1 / 2}$ with $h$ and $\lambda^{-1}$ in Table 1 was consistent with the experimental values in the experimental error, that is, less than $3 \%$. The hydrodynamic radius $R_{\mathrm{H}}$ for the wormlike cylinder was formulated by Yamakawa and Fujii. ${ }^{37,45}$ The $R_{\mathrm{H}}$ values calculated from the parameters in Table 1 are not very different from the experimental data, that is, the difference is between $0.2 \%$ and $14 \%$. This slight inconsistency is most likely because the hydrodynamic chain thickness can be different from the parameter $(d)$ as is also shown for fully substituted AEC. ${ }^{27}$ Indeed, the experimental $R_{\mathrm{H}}$ can be reproduced by the calculated value when we choose a certain possible $d$ value for each sample. 


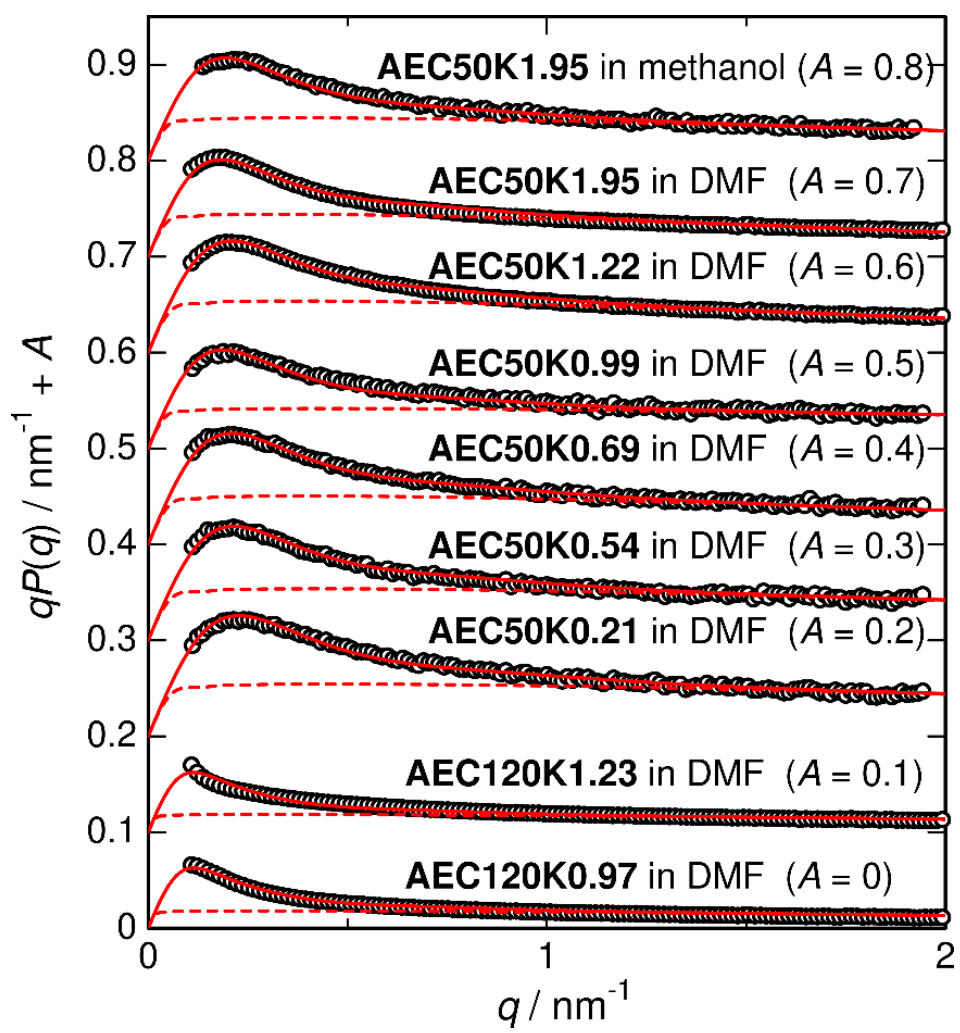

Figure 1. Holtzer plots for the indicated AEC samples in DMF or methanol at $25^{\circ} \mathrm{C}$. Solid curves, theoretical values for the touched-bead wormlike chain with the parameters in Table 1. Dashed curves are the rod limiting values $\left(\lambda^{-1}=\infty\right)$.

Figure 2 illustrates $D S$ dependence of $h$ and $\lambda^{-1}$ for AEC in DMF and methanol. The chain stiffness tends to decrease with decreasing $D S$ but still slightly larger than that for amylose in previously investigated solvents $(\sim 3 \mathrm{~nm})^{26,46-48}$ except for a metal complex solvent. ${ }^{49}$ The parameter $h$ reflecting the local helical structure also decreases with decreasing $D S$ and the lowest value is fairly consistent with amylose in an ionic liquid. ${ }^{26}$ These small $h$ values suggest that the current AEC chains have helical nature at least locally. We leave the detailed discussion of the $D S$ dependence of $h$ and $\lambda^{-1}$ because it can be caused not only by hydrogen bonding interactions described in our previous papers ${ }^{21,27,50}$ but also by the other interactions between differently substituted monomeric units. 

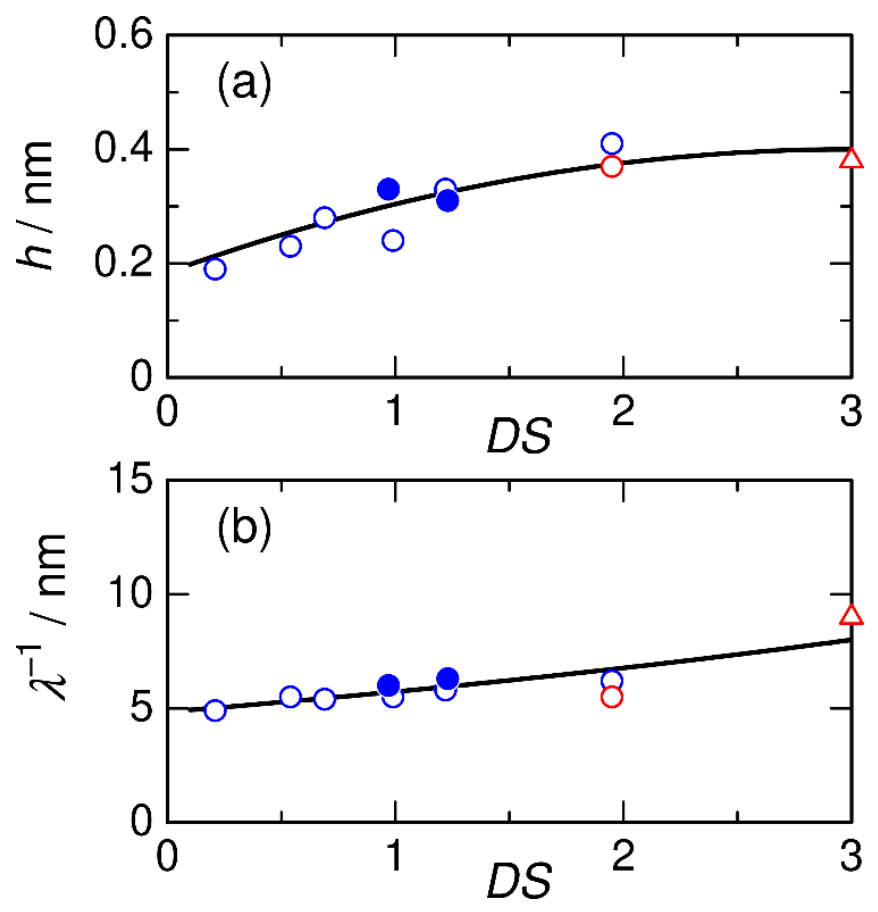

Figure 2. Degree of substitution $D S$ dependence of the helix rise per residue $h$ (a) and the Kuhn segment length $\lambda^{-1}$ (b) for AEC50K in DMF (blue unfilled circles), AEC120K in DMF (blue filled circles), AEC50k in methanol (a red unfilled circle) and the literature value ${ }^{27}$ for the fully substituted AEC in methanol (a triangle) for which $M_{\mathrm{w}}$ ranges from 10 to $1000 \mathrm{~kg} \mathrm{~mol}^{-1}$.

Conformational Properties of AEC in Water. The Holtzer plots for AEC50K0.69 shown in Figure S6 were similar to those in organic solvents in Figure 1, indicating conformational properties of AEC in aqueous solution can be modeled by the wormlike chain. A curve fitting procedure was therefore employed using eqs 1 and 2 to determine the wormlike chain parameters, $h, \lambda^{-1}$, and $d$. The theoretical solid curves reproduced the experimental data almost quantitatively. Similar analyses were also performed for AEC50K0.21 and AEC50K0.54. These three AEC samples are all soluble in water in a wide temperature range.

The obtained parameters are plotted against temperature as shown in Figure 3. The wormlike chain parameters change only gradually with temperature. The $h$ values decrease both with increasing $D S$ and raising temperature whereas $\lambda^{-1}$ does not change significantly. While the result indicates that the local helical structure in aqueous solution is similar to those in the organic solvents described above, this $h$ decreasing behavior is likely due to increasing the attractive interactions between intramolecular neighboring groups. We note that the experimental $\left\langle S^{2}\right\rangle^{1 / 2}$ values can be explained consistently with the wormlike chain parameters as in the case of the organic solvent systems. 


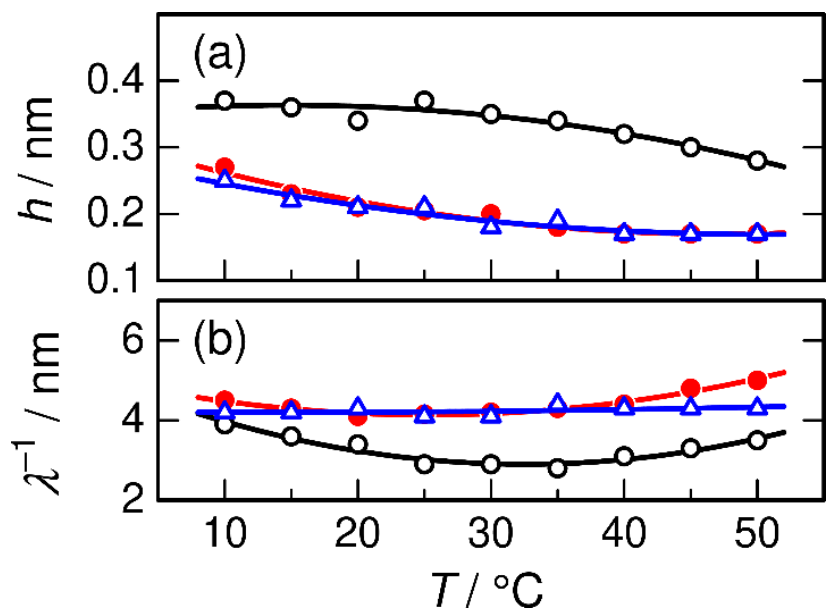

Figure 3. Temperature dependence of $h$ (a) and $\lambda^{-1}$ (b) for AEC50K0.21 (unfilled circles), AEC50K0.54 (filled circles), and AEC50K0.69 (triangles) in water.

Phase Diagram of Aqueous Solution. The transmittance of visible light suddenly decreases with raising the temperature as shown in Figure 4. The temperature at which the transmittance was reduced to $90 \%$ is defined as $T_{\text {cloud. }}$ Figure 5 illustrates the cloud point curves of the four AEC samples, AEC50K0.99, AEC120K0.97, AEC50K1.22, and AEC120K1.23 in water. The area above the cloud point is the two-phase region. Macroscopic phase separation was sometimes found in the region. This is usually found for polymers in poor organic solvent systems and poly $(N, N$-diethylacrylamide $)$ in water. ${ }^{51}$ Convex upward behavior is typical for the LCST type phase separation system and the minimum of the curve can be referred to as the critical solution point. Taking into consideration that AEC50K0.99 and AEC120K0.97 (or AEC50K1.22 and AEC120K1.23) have similar $D S$, the critical temperature $T_{\mathrm{c}}$ tends to fall with increasing molar mass. This is consistent with that the entropy of dissolution becomes lower with increasing molar mass of the polymer. On the other hand, the critical temperature significantly declines with increasing $D S$, and furthermore, the phase separation was not found for the AEC samples with $D S$ $<0.9$ in the investigated temperature range $\left(<80{ }^{\circ} \mathrm{C}\right)$. This suggests the ethylcarbamate groups behave as hydrophobic group in aqueous media. 

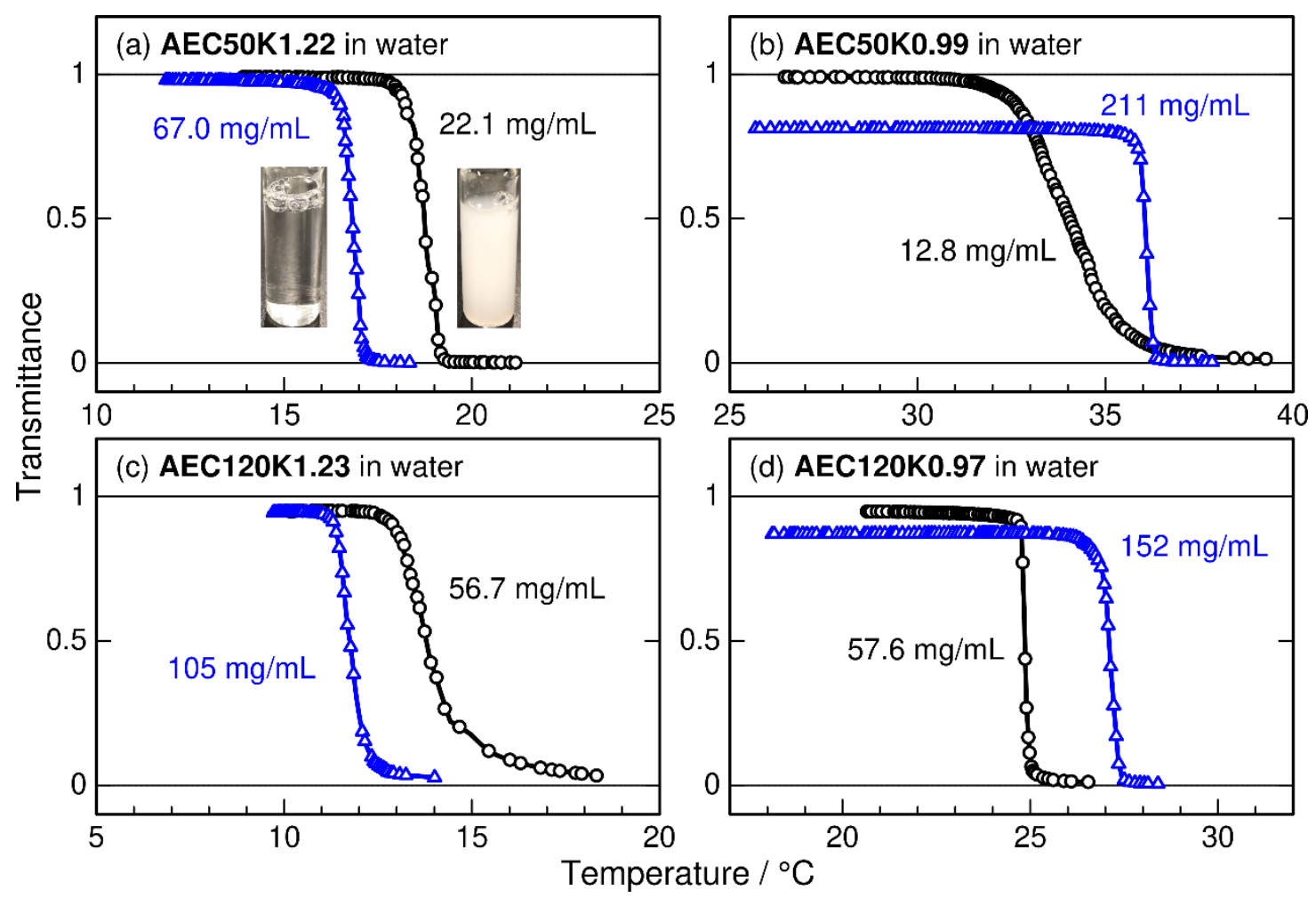

Figure 4. Typical results of the turbidity measurement for (a) AEC50K1.22, (b) AEC50K0.99, (c) AEC120K1.23, and (d) AEC120K0.97 in water. Each transmittance value was evaluated with the heating rate of $0.5^{\circ} \mathrm{C} / \mathrm{min}$.

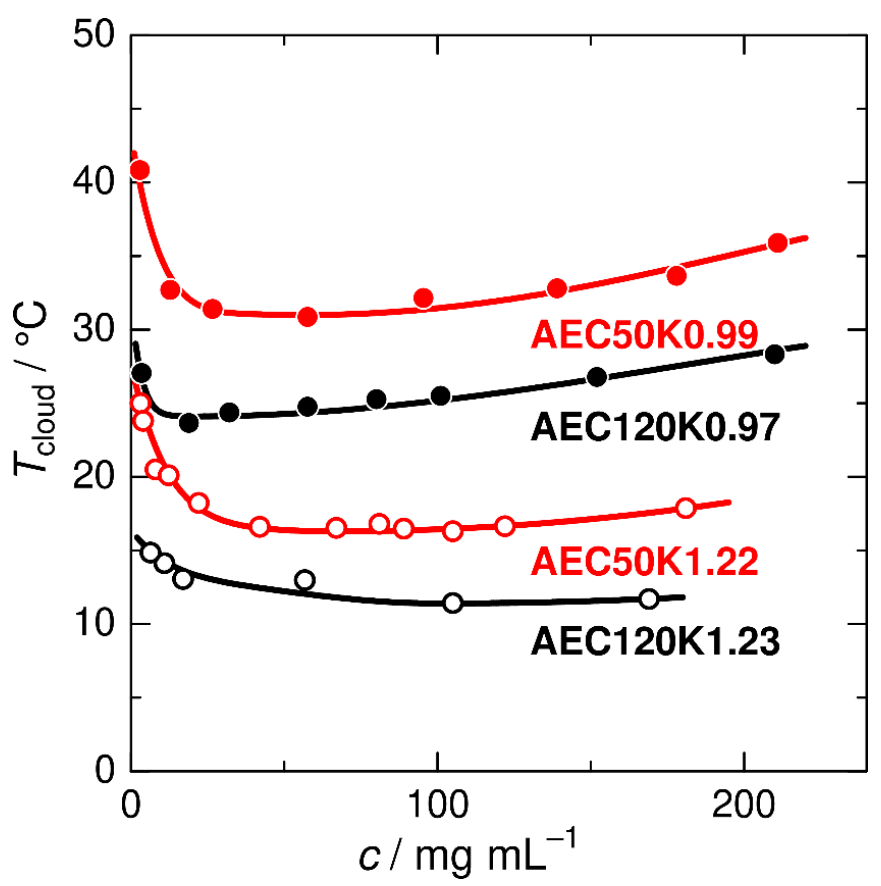

Figure 5. Cloud point curves for the indicated AEC samples in aqueous solution. 
In general, the LCST type phase separation of aqueous system is caused by the dehydration, which can be detected by the DSC measurement. The DSC curves for AEC50K0.99, AEC50K1.22, and AEC120K0.97 in water (Figure S7) had significant endothermic peaks near the cloud point temperature (vertical broken lines). Broad and asymmetric peaks are most likely due to the substituent distribution. Relatively slow decrease of the transmittance in Figure 4 supports this suggestion. Regioselectively substituted AEC is desired to discuss the endothermic behavior more quantitatively. The molar enthalpy change calculated from the area of the peak is 2.1, 1.3, and $1.7 \mathrm{~kJ} \mathrm{~mol}^{-1}$ for AEC50K0.99, AEC50K1.22, and AEC120K0.97, respectively. These values are appreciably smaller than that for PNIPAM $\left(\sim 14 \mathrm{~kJ} \mathrm{~mol}^{-1}\right){ }^{7}$ If the enthalpy change is related to the number of hydrogen bonding water molecules, the current result suggests that such water molecules on AEC is substantially fewer than that for PNIPAM.

We also attempted to find the other LCST system with another polysaccharide ethylcarbamate. While we synthesized partly substituted ethylcarbamate derivatives of cellulose, pullulan, and curdlan with substantially the same method for AEC, ${ }^{52-53}$ the cellulose derivatives $(0.2<D S<0.7)$ were not soluble in water, and furthermore, we only found completely soluble or insoluble systems both for pullulan and curdlan derivatives in water. The boundary $D S$ values were 0.6 and 0.9 for pullulan and curdlan, respectively. Namely, temperature-responsive derivatives might be found while the $D S$ range would be very narrow. If we consider the locally helical fully substituted AEC $(D S=3)$ has higher solubility than cellulose tris(ethylcarbamate) in organic solvents, ${ }^{53}$ the helical nature of AEC chains can be important to realize the good solubility even in the poor solvent, and hence, the temperature responsive behavior for $0.9<D S<1.3$ was revealed. Indeed, both UCST and LCST type theta solvent systems were also found for fully substituted amylose carbamates ${ }^{19-21}$ whereas it is rarely found for other semiflexible chain systems. ${ }^{54}$

Complex Formation Ability. One of the representative characteristics of starch and amylose is the complex formation with hydrophobic and/or amphiphilic molecules in aqueous solution. Here we show the results of iodine test to investigate the complex formation ability of AEC in aqueous solution. Figure 6 shows photographs and absorption spectra for mixed aqueous solution of the AEC samples and KI-I2. Appreciable visible light absorption was found for all AEC samples while the color gradually changes with $D S$. The UV-Vis absorption spectra in which molar extinction coefficient $\varepsilon$ of iodine plotted against $\lambda_{0}$ indicate that much higher absorbance is found in the visible light wavelength range than that without AEC. Both the wavelength and height of the absorption peak gradually decreases with increasing $D S$ and the peak height of $\varepsilon$. This change is similar to that for the molar-mass dependence of amylose $\mathrm{e}^{55}$ and the current range of the visible light absorption spectra corresponds to pure amylose with the degree of polymerization being higher than 30, indicating that all the water-soluble AEC samples have complex formation ability with iodine. 

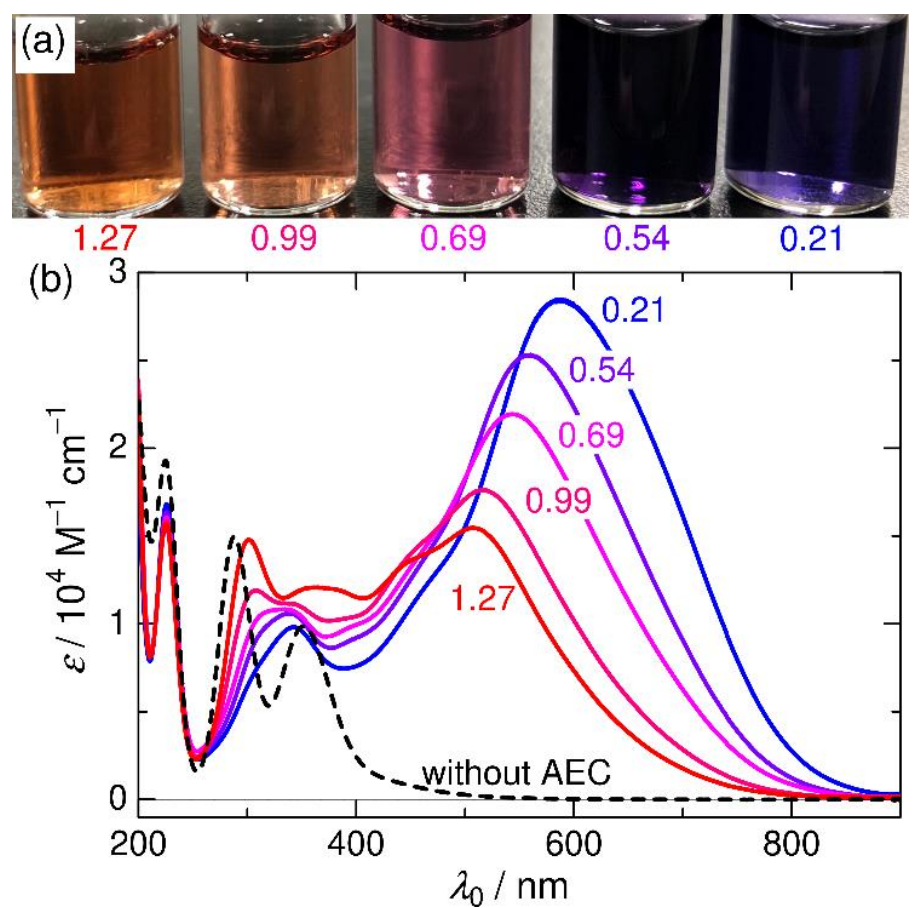

Figure 6. (a) Photo images and (b) UV-VIS absorption spectra for the AEC samples with KI-I2 solution at $25{ }^{\circ} \mathrm{C}$. Numerical symbols are the $D S$ values of each AEC sample. Dashed curve, the data without AEC. The polymer mass concentration of AEC was $5 \mathrm{mg} \mathrm{mL}^{-1}$ and the molar concentration of $\mathrm{I}_{2}$ and $\mathrm{KI}$ are both $10 \mathrm{mM}$.

The solubility of the obtained complex can be different from the original AEC in water. Figure 7 compares cloud point curves with and without $\mathrm{I}_{2}$. The cloud point temperatures of the complex is appreciably higher than that without iodine. Taking into account the negatively charged $\mathrm{I}_{3}{ }^{-}$species ${ }^{56}$ confined in the AEC molecules, intermolecular electrostatic repulsive force of the complex makes the complex more hydrophilic. According to Huang et al., ${ }^{57}$ similar behavior was also found for polymeric crown ethers with potassium ions. 


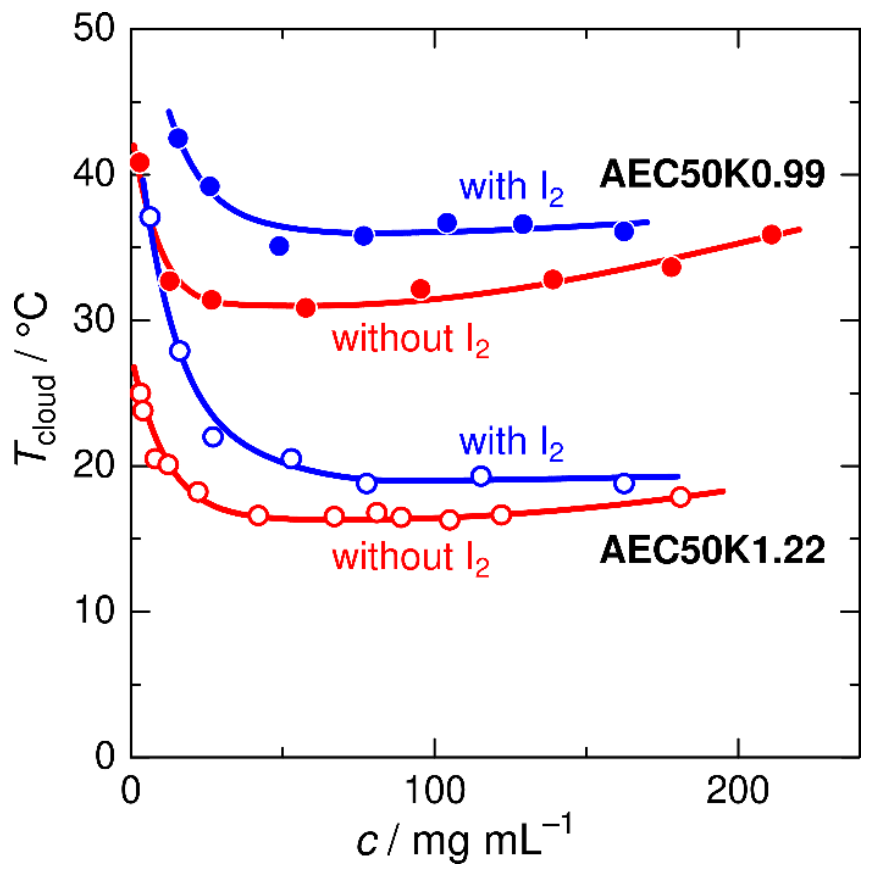

Figure 7. Cloud point curves for AEC50K0.99 (filled circles) and AEC50K1.22 with or without iodine. The molar concentration of $\mathrm{I}_{2}$ and $\mathrm{KI}$ are both $10 \mathrm{mM}$.

\section{Conclusions}

Partly substituted amylose ethylcarbamate ranging in the degree of substitution $(D S)$ between 0.2 and 1.3 are soluble in water at low temperature. The lower critical solution temperature (LCST) type phase separation behavior was found for the AEC samples with DS being above 0.9 and the LCST descents from $31^{\circ} \mathrm{C}$ to $11^{\circ} \mathrm{C}$ with increasing both $D S$ and molar mass, indicating that the LCST is tunable around the room temperature. While the chain stiffness in polar organic solvents, methanol and DMF, is typical for relatively flexible polysaccharides, the locally helical structure is recognized from the helix rise per residue. This conformational feature of AEC in water is essentially similar to those in the organic solvents. Inclusion complex with iodine was found for the all water-soluble AEC samples and the cloud point temperature of the complex is $3-7^{\circ} \mathrm{C}$ higher than the original AEC-water system, suggesting negative charge of the (poly) iodine increase the solubility. In summary, the AEC samples with $0.9<D S<1.3$ are bifunctional materials having the temperature-responsive behavior and inclusion complex formation ability in aqueous solution. Consequently, AEC can potentially be used for capturing reagent to remove lipophilic molecules from aqueous solution.

\section{Supporting Information}

A ${ }^{1} \mathrm{H}-\mathrm{NMR}$ spectrum, additional light scattering and SAXS data, and the DSC curves. This material is available free of charge via the Internet at http://pubs.acs.org. 


\section{Acknowledgments}

The authors are grateful to Prof. Takahiro Sato (Osaka University) for extensive discussion, to Mr. Akihito Kobayashi for the preliminary synthesis for curdlan ethylcarbamate samples, and to Dr. Noboru Ohta (SPring-8), Prof. Noriyuki Igarashi (KEK-PF), and Prof. Nobutaka Shimizu (KEK-PF) for SAXS measurements. The synchrotron radiation experiments were performed at the BL40B2 in SPring-8 with the approval of the Japan Synchrotron Radiation Research Institute (JASRI) (proposal nos. 2018A1124 and 2019A1072) and at the BL-6A beamline in KEK-PF under the approval of the Photon Factory Program Advisory Committee (Proposal No. 2018G519). The ${ }^{1} \mathrm{H}-\mathrm{NMR}$ measurement and the ultimate analysis were performed at the Analytical Instrument Facility, Graduate School of Science, Osaka University. This work was partly supported by JSPS KAKENHI grant numbers JP17K05884, JP18H02020, and JP20H02788.

\section{References}

1. Flory, P. J. Principles of Polymer Chemistry. Cornell University Press: Ithaca, N. Y. , 1953.

2. Yamato, M.; Akiyama, Y.; Kobayashi, J.; Yang, J.; Kikuchi, A.; Okano, T. Temperatureresponsive cell culture surfaces for regenerative medicine with cell sheet engineering. Prog. Polym. Sci. 2007, 32, 1123-1133.

3. Stuart, M. A.; Huck, W. T.; Genzer, J.; Muller, M.; Ober, C.; Stamm, M.; Sukhorukov, G. B.; Szleifer, I.; Tsukruk, V. V.; Urban, M.; Winnik, F.; Zauscher, S.; Luzinov, I.; Minko, S. Emerging applications of stimuli-responsive polymer materials. Nat. Mater. 2010, 9, 101-13.

4. Kuckling, D.; Doering, A.; Krahl, F.; Arndt, K. F. Stimuli-Responsive Polymer Systems. In Polymer Science: A Comprehensive Reference, Matyjaszewski, K.; Möller, M., Eds. Elsevier: Amsterdam, 2012; pp 377-413.

5. $\quad$ Nagase, K.; Yamato, M.; Kanazawa, H.; Okano, T. Poly(N-isopropylacrylamide)-based thermoresponsive surfaces provide new types of biomedical applications. Biomaterials 2018, $153,27-48$.

6. Ohya, Y. Temperature-responsive biodegradable injectable polymer systems with conveniently controllable properties. Polym. J. 2019, 51, 997-1005.

7. Heskins, M.; Guillet, J. E. Solution Properties of Poly(N-isopropylacrylamide). J. Macromol. Sci., Chem. 1968, 2, 1441-1455.

8. Fujishige, S.; Kubota, K.; Ando, I. Phase-Transition of Aqueous-Solutions of Poly(NIsopropylacrylamide) and Poly(N-Isopropylmethacrylamide). J. Phys. Chem. 1989, 93, 33113313.

9. Tong, Z.; Zeng, F.; Zheng, X.; Sato, T. Inverse Molecular Weight Dependence of Cloud Points for Aqueous Poly(N-isopropylacrylamide) Solutions. Macromolecules 1999, 32, 44884490.

10. Chiklis, C. K.; Grasshoff, J. M. Swelling of thin films. I. Acrylamide-Nisopropylacrylamide copolymers in water. J. Polym. Sci., Part A-2: Polym. Phys. 1970, 8, 16171626.

11. Zhang, J.; Pelton, R. The surface tension of aqueous poly(N-isopropylacrylamide-coacrylamide). J. Polym. Sci., Part A: Polym. Chem. 1999, 37, 2137-2143.

12. Hoffman, A. S.; Stayton, P. S.; Bulmus, V.; Chen, G. H.; Chen, J. P.; Cheung, C.; Chilkoti, A.; Ding, Z. L.; Dong, L. C.; Fong, R.; Lackey, C. A.; Long, C. J.; Miura, M.; Morris, 
J. E.; Murthy, N.; Nabeshima, Y.; Park, T. G.; Press, O. W.; Shimoboji, T.; Shoemaker, S.; Yang, H. J.; Monji, N.; Nowinski, R. C.; Cole, C. A.; Priest, J. H.; Harris, J. M.; Nakamae, K.; Nishino, T.; Miyata, T. Really smart bioconjugates of smart polymers and receptor proteins. $J$. Biomed. Mater. Res. 2000, 52, 577-586.

13. Shen, Z. Y.; Terao, K.; Maki, Y.; Dobashi, T.; Ma, G. H.; Yamamoto, T. Synthesis and phase behavior of aqueous poly( $\mathrm{N}$-isopropylacrylamide-co-acrylamide), poly(N-

isopropylacrylamide-co-N,N-dimethylacrylamide) and poly (N-isopropylacrylamide-co-2hydroxyethyl methacrylate). Colloid. Polym. Sci. 2006, 284, 1001-1007.

14. Hashidzume, A.; Matsumoto, A.; Mori, T.; Shikata, T.; Sato, T. Phase behavior of aqueous solutions of copolymers of N,N'-diisopropylfumaramide and N-isopropylacrylamide: effect of the density of side chains. Langmuir 2012, 28, 5522-6.

15. Heymann, E. Studies on sol-gel transformations. I. The inverse sol-gel transformation of methylcellulose in water. Trans. Faraday Soc. 1935, 31, 0846-0863.

16. Morozova, S.; Schmidt, P. W.; Bates, F. S.; Lodge, T. P. Effect of Poly(ethylene glycol) Grafting Density on Methylcellulose Fibril Formation. Macromolecules 2018.

17. Schmidt, P. W.; Morozova, S.; Owens, P. M.; Adden, R.; Li, Y. F.; Bates, F. S.; Lodge, T. P. Molecular Weight Dependence of Methylcellulose Fibrillar Networks. Macromolecules 2018, 51, 7767-7775.

18. Ju, B.; Zhang, C.; Zhang, S. Thermoresponsive starch derivates with widely tuned LCSTs by introducing short oligo(ethylene glycol) spacers. Carbohydr. Polym. 2014, 108, 307-12.

19. Burchard, W. Thermodynamic Solution Properties of 3 Polymers Exhibiting Lower Critical Solution Temperature. Polymer 1969, 10, 467-475.

20. Fujii, T.; Terao, K.; Tsuda, M.; Kitamura, S.; Norisuye, T. Solvent-Dependent Conformation of Amylose Tris(phenylcarbamate) as Deduced from Scattering and Viscosity Data. Biopolymers 2009, 91, 729-736.

21. Sano, Y.; Terao, K.; Arakawa, S.; Ohtoh, M.; Kitamura, S.; Norisuye, T. Solution properties of amylose tris(n-butylcarbamate). Helical and global conformation in alcohols. Polymer 2010, 51, 4243-4248.

22. Kong, T. F.; Guo, G. Q.; Zhang, H. T.; Gao, L. Post-synthetic modification of polyvinyl alcohol with a series of $\mathrm{N}$-alkyl-substituted carbamates towards thermo and CO2-responsive polymers. Polym. Chem. 2017, 8, 5769-5779.

23. Aburto, J.; Alric, I.; Thiebaud, S.; Borredon, E.; Bikiaris, D.; Prinos, J.; Panayiotou, C. Synthesis, characterization, and biodegradability of fatty-acid esters of amylose and starch. $J$. Appl. Polym. Sci. 1999, 74, 1440-1451.

24. Kitamura, S.; Yunokawa, H.; Mitsuie, S.; Kuge, T. Study on Polysaccharide by the Fluorescence Method .2. Micro-Brownian Motion and Conformational Change of Amylose in Aqueous-Solution. Polym. J. 1982, 14, 93-99.

25. Terao, K.; Fujii, T.; Tsuda, M.; Kitamura, S.; Norisuye, T. Solution Properties of Amylose Tris(phenylcarbamate): Local Conformation and Chain Stiffness in 1,4-Dioxane and 2Ethoxyethanol. Polym. J. 2009, 41, 201-207.

26. Jiang, X. Y.; Kitamura, S.; Sato, T.; Terao, K. Chain Dimensions and Stiffness of Cellulosic and Amylosic Chains in an Ionic Liquid: Cellulose, Amylose, and an Amylose Carbamate in BmimCl. Macromolecules 2017, 50, 3980-3985.

27. Terao, K.; Maeda, F.; Oyamada, K.; Ochiai, T.; Kitamura, S.; Sato, T. Side-chaindependent helical conformation of amylose alkylcarbamates: amylose tris(ethylcarbamate) and amylose tris(n-hexylcarbamate). J. Phys. Chem. B 2012, 116, 12714-20. 
28. Ryoki, A.; Kim, D.; Kitamura, S.; Terao, K. Linear and cyclic amylose derivatives having brush like side groups in solution: Amylose tris( $\mathrm{n}$-octadecylcarbamate)s. Polymer 2018, 137, 13-21.

29. Sirvio, J. A.; Heiskanen, J. P. Carbamation of Starch with Amine Using Dimethyl Carbonate as Coupling Agent. ACS Omega 2019, 4, 15702-15710.

30. Jinbo, Y.; Teranuma, O.; Kanao, M.; Sato, T.; Teramoto, A. Light-scattering study of semiflexible polymer solutions. 4. n-hexane solutions of poly(n-hexyl isocyanate).

Macromolecules 2003, 36, 198-203.

31. Furusawa, K.; Terao, K.; Nagasawa, N.; Yoshii, F.; Kubota, K.; Dobashi, T. Nanometersized gelatin particles prepared by means of gamma-ray irradiation. Colloid. Polym. Sci. 2004, 283, 229-233.

32. Berry, G. C. Thermodynamic and Conformational Properties of Polystyrene. I. LightScattering Studies on Dilute Solutions of Linear Polystyrenes. J. Chem. Phys. 1966, 44, 45504564.

33. Shimizu, N.; Mori, T.; Igarashi, N.; Ohta, H.; Nagatani, Y.; Kosuge, T.; Ito, K. Refurbishing of Small-Angle X-ray Scattering Beamline, BL-6A at the Photon Factory. J. Phys.: Conf. Ser. 2013, 425, 202008.

34. Shimizu, N.; Yatabe, K.; Nagatani, Y.; Saijyo, S.; Kosuge, T.; Igarashi, N. Software development for analysis of small-angle X-ray scattering data. AIP Conf. Proc. 2016, 1741, 050017.

35. Holtzer, A. Interpretation of the Angular Distribution of the Light Scattered by a Polydisperse System of Rods. J. Polym. Sci. 1955, 17, 432-434.

36. Burchard, W.; Kajiwara, K. The statistics of stiff chain molecules. I. The particle scattering factor. Proc. R. Soc. London, Ser. A 1970, 316, 185-199.

37. Yamakawa, H.; Yoshizaki, T. Helical Wormlike Chains in Polymer Solutions. Springer: Berlin, Germany, 2016.

38. Nakamura, Y.; Norisuye, T. Brush-like polymers. In Soft Matter Characterization, Borsali, R.; Pecora, R., Eds. Springer Netherlands: 2008; pp 235-286.

39. Nakamura, Y.; Norisuye, T. Scattering function for wormlike chains with finite thickness. J. Polym. Sci., Part. B: Polym. Phys. 2004, 42, 1398-1407.

40. Yamakawa, H.; Stockmayer, W. H. Statistical mechanics of wormlike chains. II. Excluded volume effects. J. Chem. Phys. 1972, 57, 2843-2854.

41. Shimada, J.; Yamakawa, H. Statistical-Mechanics of Helical Worm-Like Chains .25. Excluded-Volume Effects. J. Chem. Phys. 1986, 85, 591-600.

42. Norisuye, T.; Fujita, H. Excluded-Volume Effects in Dilute Polymer Solutions. XIII. Effects of Chain Stiffness. Polym. J. 1982, 14, 143-147.

43. Norisuye, T.; Tsuboi, A.; Teramoto, A. Remarks on Excluded-Volume Effects in Semiflexible Polymer Solutions. Polym. J. 1996, 28, 357-361.

44. Benoit, H.; Doty, P. Light Scattering from Non-Gaussian Chains. J. Phys. Chem. 1953, 57, 958-963.

45. Yamakawa, H.; Fujii, M. Translational Friction Coefficient of Wormlike Chains. Macromolecules 1973, 6, 407-415.

46. Burchard, W. Das Viskositatsverhalten Von Amylose in Verschiedenen Losungsmitteln .24. Makromol. Chem. 1963, 64, 110-125.

47. Nakanishi, Y.; Norisuye, T.; Teramoto, A.; Kitamura, S. Conformation of Amylose in Dimethyl-Sulfoxide. Macromolecules 1993, 26, 4220-4225. 
48. Norisuye, T. Viscosity Behavior and Conformation of Amylose in Various Solvents. Polym. J. 1994, 26, 1303-1307.

49. Seger, B.; Aberle, T.; Burchard, W. Solution behaviour of cellulose and amylose in ironsodiumtartrate (FeTNa). Carbohydr. Polym. 1996, 31, 105-112.

50. Terao, K.; Murashima, M.; Sano, Y.; Arakawa, S.; Kitamura, S.; Norisuye, T. Conformational, Dimensional, and Hydrodynamic Properties of Amylose Tris( $n$-butylcarbamate) in Tetrahydrofuran, Methanol, and Their Mixtures. Macromolecules 2010, 43, 1061-1068.

51. Watanabe, R.; Takaseki, K.; Katsumata, M.; Matsushita, D.; Ida, D.; Osa, M.

Characterization of poly(N,N-diethylacrylamide) and cloud points in its aqueous solutions. Polym. J. 2016, 48, 621-628.

52. Ochiai, T.; Terao, K.; Nakamura, Y.; Yoshikawa, C.; Sato, T. Rigid helical conformation of curdlan tris(phenylcarbamate) in solution. Polymer 2012, 53, 3946-3950.

53. Jiang, X. Y.; Ryoki, A.; Terao, K. Dimensional and hydrodynamic properties of cellulose tris(alkylcarbamate)s in solution: Side chain dependent conformation in tetrahydrofuran.

Polymer 2017, 112, 152-158.

54. Norisuye, T. Semiflexible Polymers in Dilute-Solution. Prog. Polym. Sci. 1993, 18, 543584.

55. Handa, T.; Yajima, H. On the blue color of triiodide ions in starch and starch fractions. II. Characterization of the changes in absorption and circular dichroism spectra of triiodide ions in amylose with the DP. Biopolymers 1980, 19, 723-740.

56. Cronan, C. L.; Schneider, F. W. Cooperativity and composition of the linear amyloseiodine-iodide complex. J. Phys. Chem. 1969, 73, 3990-4004.

57. Huang, D. C.; Zhang, Q.; Deng, Y.; Luo, Z.; Li, B.; Shen, X.; Qi, Z. H.; Dong, S. Y.; Ge, Y.; Chen, W. Polymeric crown ethers: LCST behavior in water and stimuli-responsiveness. Polym. Chem. 2018, 9, 2574-2579. 
For Table of Contents Use Only

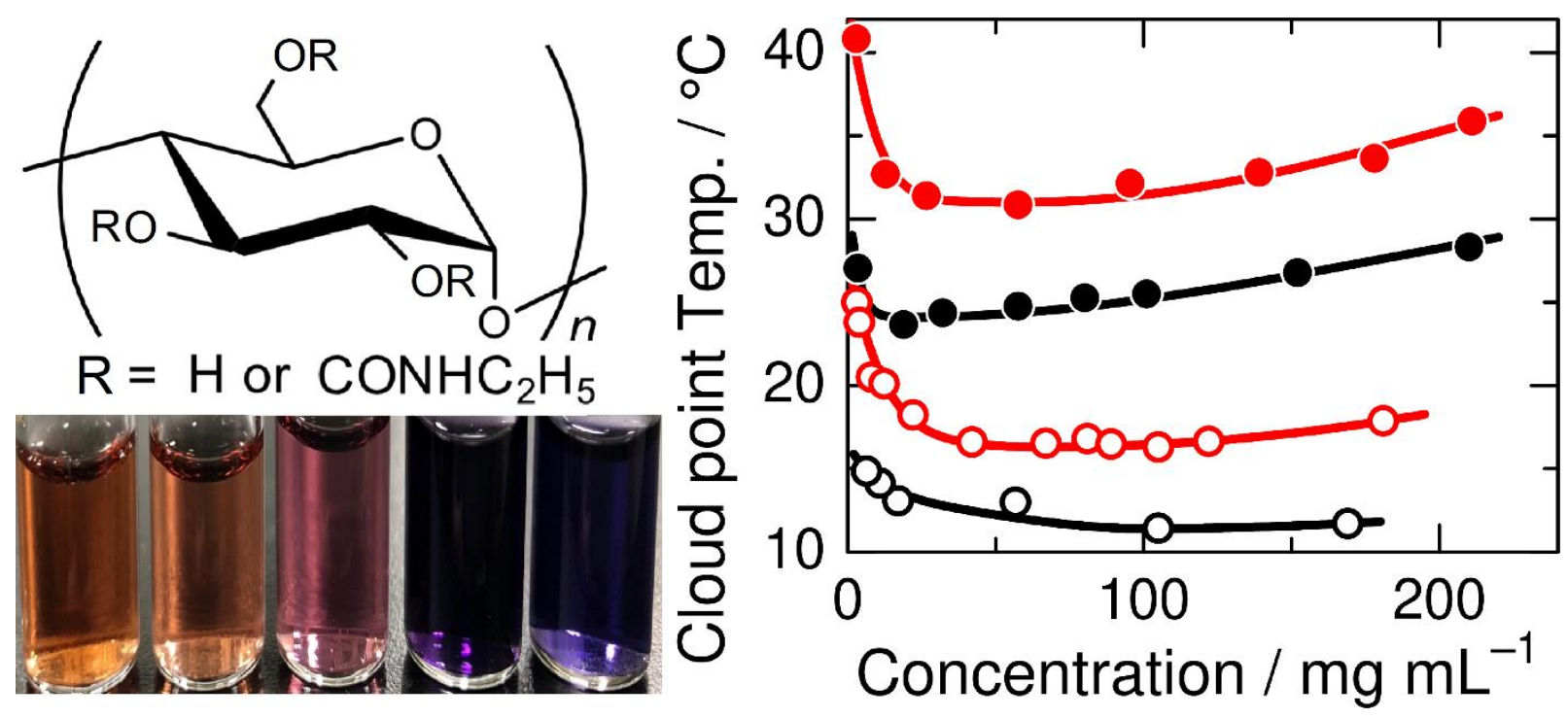

